\title{
Perspektiven der Arbeitspolitik nach der Krise: Entwicklungslinien und Handlungsbedingungen
}

In der seit Jahren intensiv geführten Debatte über Perspektiven betrieblicher Arbeitspolitik hat der Hinweis auf negative Wirkungen einer finanzmarktdominierten Unternehmenssteuerung eine große Rolle gespielt. Auf der Basis eines Überblicks über zentrale arbeitspolitische Entwicklungslinien und Einschätzungen von Betriebsräten aus der Automobilindustrie geht der Beitrag der Frage nach, inwieweit sich die Handlungsbedingungen für Arbeitspolitik im Zeichen von Krise und De-Legitimierung der Shareholder-Value-Ökonomie verändern (könnten).

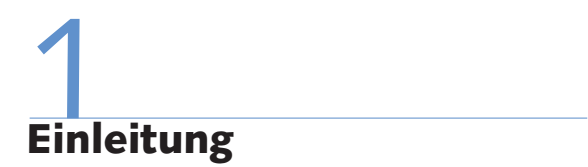

Strukturmerkmale und soziale Wirkungen des Finanzmarktkapitalismus sind seit gut einem Jahrzehnt ein Bezugspunkt sozialwissenschaftlicher Analysen der Entwicklung von Unternehmen und Erwerbsarbeit (Höpner 2003; Dörre/Röttger 2003; Windolf 2005; Froud et al. 2006). So finden sich seit Ende der 1990er Jahre bei verschiedenen Autoren Hinweise, dass die unter Überschriften wie "Shareholder Value" und „wertorientierte Unternehmensführung“ forcierte Finanzialisierung betrieblicher Strategien für das Feld der Arbeitspolitik nicht folgenlos geblieben ist. „Frißt die Shareholder-Value-Ökonomie die Modernisierung der Arbeit?" (Schumann 1998) oder „Das deutsche Produktionsmodell unter dem Druck des Shareholder Value“ (Dörre 2001a) lauten Aufsatztitel, die den Grundtenor vielfacher Beobachtungen pointieren: In der deutschen Industrie lässt sich seit Mitte der 1990er Jahre eine zunehmende Abkehr von Arbeitspolitikkonzepten beobachten, die auf aufgabenintegrative, qualifikationsorientierte Arbeitsstrukturen in Verbindung mit beteiligungsorientierten Organisations- und Arbeitseinsatzstrategien als Grundlage für innovationsorientierte Modernisierungskoalitionen zwischen Kapital und Arbeit setzen. Gerade in der Automobilindustrie, die sich einmal mehr als Taktgeber für den Wandel arbeitspolitischer Leitbilder erwiesen hat, haben sich im Gefolge von Concession Bargaining betriebliche Bündnisse eines gänzlich anderen $\mathrm{Zu}$ schnitts ausgebreitet, in denen es im Kern um den Tausch von Beschäftigungssicherheit gegen Zugeständnisse in den Bereichen Bezahlung, Arbeitszeiten und Leistungsre- gulierung geht (Rehder 2003). Ein Überblick über kontrollierte Abweichungen vom Flächentarifvertrag in der Metallund Elektroindustrie, wie sie das Pforzheimer Abkommen ermöglicht hat, liegt mittlerweile vor (Haipeter 2009). Eine systematische Bilanz der arbeitsbezogenen Veränderungen (Arbeitsstrukturen, Arbeitseinsatzstrategien) innerhalb der letzten Jahre steht dagegen noch aus und scheint angesichts der zunehmenden Vielfalt betrieblicher Entwicklungstendenzen (Schumann 2008) und des lückenhaften Stands der arbeits- und industriesoziologischen Forschung derzeit auch kaum möglich.

Die arbeitspolitische Debatte der letzten Jahre kreiste im Kern um die Frage, inwieweit angesichts der sich immer stärker verallgemeinernden Finanzmarktorientierung und der hiermit verbundenen Steigerung von Renditeerwartungen überhaupt noch Spielräume für innovative arbeitspolitische Konzepte bestehen, die Wirtschaftlichkeitsvorteile für die Unternehmen mit Elementen ,guter Arbeit" verbinden (Detje et al. 2005). Im Augenblick deutet sich vor dem Hintergrund der aktuell von politischen Beobachtern diagnostizierten De-Legitimierung des Finanzmarktkapitalismus eine Diskursverschiebung an. Ähnlich wie sich auf der Ebene der Makroökonomie eine Abkehr von neo-liberalen Politikkonzepten abzeichnet, stellt sich auch für den Bereich der Corporate Governance und der betrieblichen Arbeitspolitik die Frage, ob die im Bereich der Finanzökonomie ausgelöste und tief in die Realökonomie hineinreichende Krise eine erneute Zeitenwende einleiten könnte (Pickshaus/Urban 2009). Auch wenn die Zusammenhänge zwischen Finanzmarktdominanz und betrieblicher Arbeitspolitik in der arbeitsbezogenen Forschung weitgehend ungeklärt sind: Als mögliche Entwicklungsrichtung ganz ausschließen lässt es sich nicht, dass die - auch von Unternehmensvertretern verstärkt artikulierte Kritik an der Dominanz von Finanzmarktorientierungen in eine Abkehr von Shareholder Value dominierten Unternehmensleitbildern mündet und stattdessen realwirtschaftliche Kalküle und produktionsökonomische Überlegungen wieder an Bedeutung gewinnen.

Angesichts fehlender Befunde zum Verhältnis von Finanzmarktkapitalismus und Arbeitspolitik können die in der gegenwärtigen Debatte aufgeworfenen Fragen derzeit allerdings nicht seriös beantwortet werden. Der vorliegende Text konzentriert sich vielmehr darauf, in einem ersten Schritt zunächst einige grundlegende Trendbestimmungen der arbeits- und industriesoziologischen Forschung zur Entwicklung industrieller Arbeit zu skizzieren (Abschnitt 2), bevor dann, gestützt auf Interviews mit Betriebsräten aus der deutschen Automobilindustrie, ${ }^{1}$ Aussagen zu aktuellen Entwick-

\footnotetext{
Materialbasis des Beitrages sind rund 30 ausführliche Interviews mit Betriebsräten der deutschen Automobilindustrie (Endhersteller und Zulieferer), die ganz überwiegend im ersten Halbjahr 2009 durchgeführt worden sind. Bei den Gesprächspartnern handelte es sich um Arbeitspolitik- und Arbeitsgestaltungsexperten. Einbezogen waren nicht nur verschiedene Werke sämtlicher deutscher Endhersteller im PKW-Bereich, sondern auch Vertreter des Nutzfahrzeugsegmentes; bei den Zulieferunternehmen handelte es sich um eine Mischung aus kleineren Unternehmen und Mega-Zulieferern. Ein großer Teil der Interviews wurde von Heiko Spieker und Ulrich Voskamp geführt. Ermöglicht wurden die Erhebungen durch eine finanzielle Förderung der Hans-Böckler-Stiftung.
}

Martin Kuhlmann, Dr., Soziologe, Mitarbeiter am Soziologischen Forschungsinstitut (SOFI) an der Universität Göttingen. Arbeitsschwerpunkte: Industrie- und Arbeitssoziologie, Industrielle Beziehungen. e-mail: martin.kuhlmann@sofi.uni-goettingen.de 
lungsdynamiken von Arbeit und Handlungsbedingungen von Arbeitspolitik getroffen werden (Abschnitt 3). Der Begriff Arbeitspolitik zielt auf den Gegenstandsbereich der betrieblichen Arbeits- und Organisationsstrukturen, wobei jedoch ein breites Verständnis unterlegt ist, das über die Ebene der Arbeitsgestaltung und der Arbeitseinsatzkonzepte hinausgeht und betriebsorganisatorische Aspekte, die betrieblichen Führungsstrukturen und Steuerungsformen, das Thema Leistungspolitik sowie die Frage nach der Ausgestaltung von Prozessoptimierungsaktivitäten einbezieht. Ausgehend von zentralen Befunden $\mathrm{zu}$ diesen Themen werden abschließend, thesenartig verdichtet, Aussagen zu den Perspektiven von Arbeitspolitik formuliert (Abschnitt 4).

\section{2 \\ Trendbestimmungen der Entwicklung von Arbeit}

In der arbeitssoziologischen Forschung werden seit einer Reihe von Jahren divergierende Tendenzen der Entwicklung von Arbeit diagnostiziert. $\mathrm{Zu}$ nennen sind:

\subsection{RETAYLORISIERUNG}

In den 1980er und bis zu Beginn der 1990er Jahre wurden Trends des Wandels von Industriearbeit unter Stichworten wie Entdifferenzierung der Arbeitsorganisation, Rücknahme fachlicher und funktionaler Arbeitsteilung, ganzheitliche Nutzung des Arbeitsvermögens und Reprofessionalisierung gefasst (Kern/Schumann 1984; Pries et al. 1990). In den 1990er Jahren war im Kontext der ersten Lean-Production-Welle dann zunächst eine sprunghafte Zunahme von Gruppenarbeit zu verzeichnen, die über die fachliche Aufwertung von Tätigkeiten hinaus auch eine Institutionalisierung von Gruppenselbstorganisation und erweiterte Beteiligungsformen beinhaltete. Ab Ende der 1990er Jahre wendete sich jedoch das Blatt: Tendenzen einer Retaylorisierung von Arbeit wurden diagnostiziert (IG Metall 2001; Dörre 2001b; Kuhlmann 2004) - und mittlerweile sogar als industriepolitische Strategie offensiv propagiert (Gryglewski 2007). Überraschend kam dieser Rollback nicht. Als Folge der immer engeren Aus- richtung an Lean-Production-Prinzipien und Übernahme von Elementen des Toyota-Produktionssystems im Verlauf der 1990er Jahre (Jürgens 1997; Liker 2004) bildete sich eine immer stärkere Orientierung an einem durch kurzzyklisches, repetitives Arbeiten geprägten Leitbild und eine möglichst weitgehende Standardisierung von Arbeits- und Produktionsprozessen heraus. Zeitlich etwas verschoben, aber letztlich parallel zur produktionsstrukturellen Forcierung von Fließfertigungsprinzipien und arbeitsstrukturellen Retaylorisierungstendenzen, zeigten sich auf der betriebsorganisatorischen Ebene, insbesondere in der Automobilindustrie, zunächst Tendenzen eines Ausbaus zentraler Planungsprozesse. Anschließend erfolgten eine insgesamt stärkere Zentralisierung von Fachfunktionen sowie eine Rehierarchisierung der betrieblichen Führungsstrukturen.

\subsection{VERMARKTLICHUNG}

Als „Vermarktlichung“ werden in der Debatte zur Entwicklung von Arbeit im Detail durchaus unterschiedliche Organisationskonzepte gefasst (Moldaschl/ Sauer 2000), die allesamt jedoch in einem Zusammenhang zur Ausweitung von Finanzmarktorientierungen gesehen werden (Ezzamel et al. 2008). Als gemeinsames Kennzeichen spielen marktliche Elemente bei der Organisation von Arbeit eine zunehmende Rolle. Sie konfrontieren die Beschäftigten mit Marktdruck und führen in Kombination mit erweiterten Handlungsspielräumen zu erhöhtem Leistungsdruck und einer Internalisierung von Marktanforderungen (Peters/Sauer 2005). Allerdings erfolgt die Bezugnahme auf "Markt" in den unter der Überschrift „Vermarktlichung" zusammengefassten Organisationskonzepten höchst unterschiedlich (Kuhlmann 2004, S. 316ff.): Als radikale Vermarktlichung (Ausgliederung in den Markt) lassen sich die bereits in verschiedenen Dezentralisierungsstudien erforschten Formen der Ausgründung und Verselbstständigung von Organisationseinheiten bezeichnen, sofern es hierbei zu Leistungsvereinbarungen auf der Basis frei verhandelbarer Verrechnungspreise kommt. Im Mittelpunkt der Debatte stehen jedoch verschiedene Formen einer hybriden Vermarktlichung, die sich zumeist durch eine Kombination von marktförmigen oder marktnahen Koordina- tionsformen mit hierarchischen Steuerungsprinzipien auszeichnen. Hier wäre zum einen die Koordination durch Markt zu nennen, worunter insbesondere Praktiken der Koordination über interne Leistungsvereinbarungen auf der Basis von mikropolitisch beeinflussten - Verrechnungspreisen fallen. Aber auch der seit den 1990er Jahren in Großunternehmen immer häufiger praktizierte Standortwettbewerb bei der Vergabe von Produktionsaufträgen und Investitionen zählt hierzu. Zum anderen lassen sich auch Formen einer engeren Steuerung am Markt als Vermarktlichung bezeichnen. Letztere reichen von einer engeren Anbindung von Organisationseinheiten an Kundenwünsche, über Zielkostensysteme (Target Costing), bei denen es bereits in frühen Phasen der Produktplanung zu einer verbindlichen Festlegung von Einzelkosten - ausgehend von Marktpreisen und Erlöszielen - kommt, bis hin zur Steuerung über Kennzahlensysteme und Zielvereinbarungen. Für die unterschiedlichen Formen der Vermarktlichung gilt, dass sie zunächst als Gegenbewegung zu zentralistisch-hierarchischen Organisationskonzepten propagiert wurden, zunehmend aber auch in Kombination mit Rezentralisierungsprozessen angewandt werden.

\subsection{SUBJEKTIVIERUNG}

Der Begriff „Subjektivierung“ zielt im Kern auf Entwicklungen, bei denen Betriebe im Gegensatz zu tayloristischen Konzepten auf eine aktive Einbindung der Beschäftigten in die Gestaltung der Arbeitsabläufe setzen. Für die Beschäftigten bestehen damit erweiterte Möglichkeiten, aber auch Anforderungen, an selbstorganisiertes Arbeiten (Kleemann et al. 2003; Lohr 2003). Unter der Überschrift „normative Subjektivierung“ werden zudem Trends diagnostiziert, wonach Beschäftigte ihrerseits erweiterte Ansprüche an Eigenständigkeit, Sinnhaftigkeit und Selbstverwirklichung in der Arbeit formulieren und als Maßstab bei der Berufswahl und Bewertung von Tätigkeiten ins Spiel bringen (Baethge 1991). Während Subjektivierungsprozesse, teilweise über professionelle Berufskulturen vermittelt, bei hochqualifizierten Tätigkeiten und Angestelltenarbeit immer schon zum Bestandteil betrieblicher Organisationsstrategien und zum Arbeitsverständnis dieser Beschäftig- 
tengruppen gehörten, ${ }^{2}$ haben neuere Organisationskonzepte ${ }^{3} \mathrm{zu}$ einer stärkeren Verbreitung von Subjektivierungstendenzen geführt. Prozesse der Subjektivierung von Arbeit greifen durchaus Ansprüche der Beschäftigten auf und beziehen sich in Teilen auf Arbeitsgestaltungsansätze wie sie auch Konzepten "guter Arbeit" unterlegt sind. Empirische Studien weisen jedoch zugleich darauf hin, dass subjektivierte Arbeitsformen in bestimmten organisatorischen Arrangements und bei bestimmten Beschäftigtengruppen, insbesondere dort, wo Arbeitskulturen und Praktiken der Leistungsregulierung wenig verbreitet sind, leicht in (Selbst-) Überforderungstendenzen einmüden können.

\subsection{PREKARISIERUNG}

Als weiterer Haupttrend der Entwicklung von Arbeit und als möglicher Bezugspunkt für arbeitspolitische Strategien der Gewerkschaften lassen sich Prozesse der Prekarisierung bezeichnen (Dörre 2005). Sie haben sich nicht zuletzt auf der Basis von schrittweisen und seit Ende der 1990er Jahre beschleunigten Veränderungen im Bereich der Arbeitsmarktgesetzgebung vollzogen (Brinkmann et al. 2006; Castel/Dörre 2009). Anders als die bisher genannten Trends haben Prozesse der Prekarisierung von Arbeit ihren Ausgangspunkt zwar nicht in arbeitsorganisatorischen Strategien. Sie sind vielmehr im Kern Bestandteil betrieblicher Flexibilisierungs- und Kostensenkungsstrategien, die am Beschäftigungsverhältnis ansetzen. Es ist jedoch davon auszugehen, dass von der Zunahme prekärer Beschäftigungsformen (insbesondere Leiharbeit, Befristungen und Niedriglohnbeschäftigung) nicht nur Verunsicherungseffekte und leistungspolitische Disziplinierungswirkungen ausgehen, sondern dass Prozesse der Prekarisierung letztlich auch betriebliche Arbeitseinsatzstrategien und Arbeitsstrukturen verändern. In einer Reihe von Branchen - insbesondere im Dienstleistungsbereich - finden sich Hinweise auf einen beschleunigten Wandel des deutschen Beschäftigungsmodells. Das wirft die Frage auf, inwieweit auch in den exportorientierten Kernindustrien das deutsche Produktionsmodell mit seiner Kombination aus Innovationsorientierung auf der Basis hochwertiger Produkte, qualifizierter Arbeit und regulierter Hochlohnbeschäftigung, ermöglicht durch vertretungsstarke Gewerkschaften, unter den
Druck einer fortschreitenden Prekarisierung von Arbeit gerät (Lehndorff 2009; Streeck 2009).

\subsection{INNOVATIVE ARBEITSPOLITIK}

Der Begriff „innovative Arbeitspolitik“ fasst schließlich Konzepte der Gestaltung von Arbeits- und Organisationsstrukturen zusammen, die an die Modernisierung des deutschen Produktionsmodells in den 1980er Jahren anknüpfen, diesen Pfad ausbauen und auf qualifizierte Aufgabenzuschnitte, verbesserte Kooperationsbeziehungen und ein höheres Maß an Selbstorganisation und Beteiligung setzen (Kuhlmann et al. 2004). Innovative Arbeitspolitik steht nicht für einen breitflächig zu beobachtenden Entwicklungstrend, wohl aber für ein zukunftsweisendes Leitbild der Organisation von Arbeit. Zentrales Bestimmungsmoment derartiger Strategien ist die Verfolgung einer Mutual-Gains-Perspektive, bei der Wirtschaftlichkeitsvorteile für die Betriebe mit Arbeitsverbesserungen aus Sicht der Beschäftigten kombiniert werden. Vorliegende Untersuchungsbefunde deuten darauf hin, dass sowohl die Wirtschaftlichkeitsverbesserungen als auch die Vorteile für die Beschäftigten sich allerdings nur in dem Maße realisieren lassen, wie die Reorganisationsansätze über den Bereich der unmittelbaren Produktionsarbeit und die Ebene der Arbeitsorganisation hinausgehen. Notwendig ist es, dabei unter anderem zu einer Neugestaltung von betriebsorganisatorischen Strukturen in Richtung einer prozessorientierten Dezentralisierung und einer Ausrichtung der betrieblichen Steuerungs- und Koordinationsformen im Sinne kooperativer Selbstorganisation zu kommen (Schumann et al. 2006; Kuhlmann 2007). Während die arbeitspolitischen Debatten der 1990er Jahre insbesondere um die Ausgestaltung von Gruppenarbeit kreisten, dürften sich für die weitere Entwicklung innovativer Arbeitspolitik Themen wie die Verbesserung von funktions- und hierarchieübergreifenden Kooperations- und Abstimmungsprozessen sowie die aktive Einbeziehung der Beschäftigten in Prozessoptimierungsaktivitäten und betriebliche Planungsprozesse als sehr viel folgenreicher erweisen. Die im Leitbild „innovative Arbeitspolitik“ verfolgten Reorganisationskonzepte stehen insofern zwar in der Kontinuität von Grundelementen des deutschen Produktionsmodells (Schumann et al. 2008), sie stellen ande- rerseits jedoch eine (notwendige) Weiterentwicklung angesichts wichtiger, bereits in den 1990er Jahren diagnostizierter Schwächen (Jürgens/Naschold 1994; Kern/ Sabel 1994) dieses Modells dar.

\subsection{ZWISCHENBILANZ: PLURALISIE- RUNG UND AMALGAMIERUNG}

Retaylorisierung, Vermarktlichung, Subjektivierung, Prekarisierung, aber auch innovative Arbeitspolitik benennen wichtige, von der Arbeits- und Industriesoziologie in den letzten Jahren diagnostizierte arbeitspolitische Entwicklungslinien. Ein verlässlicher, empirisch fundierter Überblick über die relative Verbreitung dieser Trends ist derzeit allerdings nicht möglich. Für einzelne Branchen und Tätigkeitsfelder lassen sich jedoch Gewichtungen vornehmen: So finden sich Retaylorisierungskonzepte insbesondere in manuellen Großserienfertigungsbereichen - aber eben auch in vielen Dienstleistungsbereichen oder, in Form von Standardisierungstendenzen, selbst bei vergleichsweise qualifizierten Angestelltentätigkeiten; Subjektivierung bleibt eine wichtige arbeitspolitische Strategie im Bereich mittlerer und hochqualifizierter Beschäftigungsgruppen. Sie wird vielfach jedoch mit Vermarktlichungsansätzen kombiniert; und auch Prekarisierungsprozesse sind selbst für gut qualifizierte Beschäftigtengruppen keine Seltenheit mehr (Vester et al. 2007). Bei Fallstudien in Betrieben, die eine auf innovative Arbeitspolitik ausgerichtete Linie verfolgen, zeigt sich häufig, dass Unternehmen von einer systematischen, kohärenten Ausrichtung ihrer Gestaltungsansätze über unterschiedliche Handlungsfelder hinweg weit entfernt

2 Empirische Studien der letzten Jahre deuten eher auf gegenläufige Tendenzen hin. Parallel zu der in vielen Führungskräftestudien gemachten Beobachtung, dass die Reorganisationswellen der 1990er Jahre die bei dieser Beschäftigtengruppe ausgeprägte "Beitragsorientierung " bedrohten (Kotthoff/Wagner 2008), geraten die im Zuge verstärkter Finanzmarktorientierungen erzeugten und in die verschiedenen Unternehmensbereiche und Tätigkeitsfelder hinein vermittelten Anforderungen an steigende Renditen und der hiermit einhergehende Ökonomisierungsdruck vielfach in einen Gegensatz zu professionellen Arbeitsorientierungen (Vester et al. 2007).

3 Hierzu zählen selbst organisierte Gruppenarbeit (Gerst 1998; Kuhlmann/Schumann 2000), die Zunahme projektförmiger Arbeitsstrukturen, aber auch leistungspolitische Instrumente wie variable Formen der Bezahlung, Zielvereinbarungen oder Vertrauensarbeitszeit. 
sind. Im Regelfall mischen sich beispielsweise Elemente einer innovativen Arbeitspolitik - etwa bei der Gestaltung von Gruppenarbeit - mit Retaylorisierungs- oder Vermarktlichungsphänomenen. Besonders häufig anzutreffen ist eine Kombination verschiedener, auch entgegengesetzter Leitbilder: Einerseits werden die qualifikatorischen und motivationalen Kompetenzen und die Kooperationsfähigkeit der Beschäftigten betont; andererseits finden sich gerade im Bereich der Leistungspolitik und der Beschäftigungsbedingungen Formen einer rigiden Ökonomisierung (Kratzer et al. 2008; Schumann 2008). Gerade weil verschiedene Dynamiken zunehmend auch in Kombinationen zu beobachten sind, werden eine arbeitspolitische Standortbestimmung und der Entwurf von Perspektiven gewerkschaftlicher Arbeitspolitik erschwert. Eine Zuordnung von arbeitspolitischen Strategien etwa zu unterschiedlichen Tätigkeitsfeldern oder Beschäftigtengruppen ist nur begrenzt möglich. Verdichten lässt sich der gegenwärtige Wissensstand eher entlang von Formeln wie Pluralisierung und Amalgamierung der arbeitspolitischen Konzepte und Strategien. Betriebliche Reorganisationsstrategien werden hinsichtlich ihrer Verursachungsbedingungen, aufgrund der vielfältigen Wechselwirkungen sowie im Hinblick auf ihre (Arbeits-)Wirkungen komplexer, widersprüchlicher und letztlich instabiler. Selbst innerhalb von Teilbranchen lassen sich aber auch erhebliche Unterschiede in den Arbeitspolitikstrategien von Unternehmen und Betrieben feststellen. Kohärente Unternehmensstrategien stellen eher die Ausnahme dar, was angesichts managementsoziologischer Einsichten in die Ungewissheit, mikropolitische Umkämpftheit und Konkurrenzhaftigkeit von Managementhandeln letztlich auch nicht verwundert.

\section{Arbeitspolitische Heraus- forderungen aus Sicht von Betriebsräten in der Automobilindustrie}

Am Beispiel der Automobilindustrie ließen sich die skizzierten Entwicklungslinien konkretisieren und differenzieren. Stattdessen sollen jetzt jedoch - auf Basis der Aussagen von Betriebsräten - in kompri- mierter Form einige Einschätzungen zu den Perspektiven von Arbeitspolitik vor dem Hintergrund der gegenwärtigen Krise formuliert werden. Die Wirkungen der Krise bestimmen in hohem Maße den Alltag der betrieblichen Interessenvertretungen, der sich insbesondere um die Ausgestaltung von Kurzarbeit dreht. Bei der Suche nach zukünftigen arbeitspolitischen Perspektiven spielt die Krise jedoch eine vergleichsweise geringe Rolle. Kurzfristig registriert die überwiegende Mehrheit der Gesprächspartner eher eine noch weitere Ressourcenverknappung für arbeitspolitische Initiativen: So werden beispielsweise Beschäftigungsüberhänge nicht für Qualifizierungsmaßnahmen genutzt; im Rahmen von Gruppenarbeit geplante Optimierungsaktivitäten fallen verschärften Sparauflagen zum Opfer. Und auch für die Zeit nach der Krise rechnen die von uns befragten Betriebsräte eher mit einem sich weiter verschärfenden Kostensenkungsdruck wie auch einer fortschreitenden Globalisierung und Intensivierung des Wettbewerbs. Für die betriebliche Arbeitspolitik dürften sich aus der Debatte über die Krise des Finanzmarkt-Kapitalismus vorerst auch deshalb kaum Impulse ergeben, weil insbesondere die Retraditionalisierungstendenzen im Bereich der Arbeits- und Organisationsgestaltung sich mit dem ToyotaProduktionssystem gerade nicht auf ein Unternehmen mit ausgeprägter Finanzmarktorientierung beziehen, sondern auf ein produktionsökonomisch ausgerichtetes Leitbild der Gestaltung von Arbeit.

\subsection{ARBEITSPOLITIK UNTER DRUCK - ABER OHNE WIRKLICHE ALTERNATIVE}

Die von uns befragten Betriebsräte sehen die betrieblichen Interessenvertretungen bei ihrem Engagement für qualifizierte Arbeit und auskömmliche Leistungsbedingungen seit Jahren in der Defensive. Selbst in gewerkschaftlich gut organisierten Betrieben mit konflikterprobten Belegschaften hat es seit etwa Mitte der 1990er Jahre von der Managementseite her nicht nur eine Politik des Concession Bargaining zur Standortsicherung gegeben, in der übertarifliche Entgeltbestandteile und leistungspolitische Regelungen (bei Pausen, der Bestimmung von Leistungsvorgaben, der Personaldichte) zulasten der Beschäftigten verändert wurden. Rückschritte sind in nahezu allen Betrieben auch im Bereich der Arbeitspolitik im engeren Sin- ne zu verzeichnen: In den Produktionsbereichen sind entlastende Umfeldaufgaben und Vormontagen teilweise entfallen; Taktzeiten, Taktausgleiche und Arbeitsumfänge wurden reduziert. Die in einigen Betrieben $\mathrm{zu}$ beobachtenden Bemühungen für eine erweiterte und systematischere Rotation kompensieren die vielfach beklagte Tendenz der Arbeitsverdichtung nur unzureichend, zumal personalpolitische Strategien der Flexibilisierung des Personaleinsatzes und steigende Anteile von Beschäftigten mit Einsatzeinschränkungen Rotationsmöglichkeiten zugleich einschränken. Auch bezogen auf die leistungspolitisch nach wie vor wenig regulierten Angestelltenbereiche wird eine erhebliche Leistungsverdichtung beklagt. Ausscheidende Beschäftigte werden nicht ersetzt, Arbeitsaufgaben werden ohne gleichzeitige Personalaufstockung ausgeweitet oder es kommt im Rahmen von Reorganisationsmaßnahmen zu einer Verlagerung von Aufgaben und Zuständigkeiten. Kritik wird außerdem an Tendenzen der Formalisierung und Standardisierung von Tätigkeiten und Abläufen geübt, die mittlerweile in nahezu allen Bereichen verstärkt zu beobachten sind. Rückschrittliche Entwicklungen werden von der überwiegenden Mehrheit der Betriebsräte schließlich auch insofern gesehen, als Beteiligungsansätze, insbesondere im Bereich der Prozessoptimierung, durch stärker hierarchiebetonte und expertengetriebene Konzepte ersetzt werden oder Beteiligung nur verengt erfolgt bei kurzfristig rechenbaren Kosteneinsparungen.

Auch wenn Betriebsräte in der überwiegenden Zahl der Betriebe von Gegenwind für Formen innovativer Arbeitspolitik berichten und außerdem monieren, dass ihnen auf der Managementseite (insbesondere auf den oberen Führungsebenen) zunehmend aktive Bündnispartner fehlen, halten sie unter normativen Gesichtspunkten an qualifikationsorientierten und beteiligungsorientierten Leitbildern fest. Sie sehen darin auch über die Krise hinaus einen tragfähigen arbeitspolitischen Ansatz und verweisen darauf, dass eine hierauf ausgerichtete Politik von den Beschäftigten unverändert eingefordert wird und auch für die Betriebe vorteilhaft wäre. In einigen Unternehmen lässt sich bei unseren Gesprächspartnern mittlerweile zwar ein gehöriges Maß an Frustration bis hin zu Resignation feststellen. Fast alle sind sich jedoch darin einig, dass innovative arbeitspolitische Konzepte 
bislang noch keineswegs ausgereizt wurden. Ihr Interesse an diesen Ansätzen ist nach wie vor hoch.

\subsection{ERHEBLICHE UNTERSCHIEDE BEI DEN ARBEITSPOLITISCHEN PRAKTIKEN}

Auch wenn als Leitbild der Gestaltung von Arbeit seit Jahren eine eher tayloristische Variante des Toyota-Produktionssystems (Jürgens 2007) überaus einflussreich ist, sind die betrieblichen Arbeitsstrukturen und Arbeitseinsatzformen durch eine erhebliche Spannbreite und relevante Unterschiede geprägt. Selbst innerhalb von Unternehmen und teilweise sogar größeren Betrieben finden sich verschiedene arbeitspolitische Orientierungen und Praktiken. Produktionsstrukturell haben sich in den Großserienmontagen und bei verbleibenden Maschinenbedienungstätigkeiten nahezu überall kurzgetaktete Fließfertigungsprozesse durchgesetzt. Und auch die Logistikprozesse sind durchweg auf das Prinzip der Minimierung von Lagerbeständen ausgerichtet, sodass sich die Anteile indirekter Tätigkeiten deutlich reduziert haben. Insbesondere in den quantitativ ebenfalls bedeutsamen technisierten Fertigungsbereichen, bei Kleinserien und im Nutzfahrzeugbereich sind die Arbeitsumfänge und die Anteile indirekter Tätigkeiten aber immer noch deutlich größer. In solchen Fertigungsbereichen spielen arbeitspolitische Konzepte, die auf Facharbeit, große Handlungs- und Entscheidungsspielräume sowie erweiterte Funktionsintegration setzen, aus Gründen der Prozessbeherrschung fast überall eine große Rolle.

Gruppenarbeit bleibt - auch aus Sicht der Beschäftigten - ein wichtiger Anspruch und ein zentraler Bezugspunkt von „guter Arbeit“. Sie verspricht verbesserte Zusammenarbeit, wechselseitige Unterstützung und hat - u.a. mit der Wahl von Gruppensprechern - demokratisierende Elemente. Allerdings hat die Qualität der Gruppenarbeit in vielen Betrieben gelitten. Nicht nur, weil es Stagnation und häufig sogar Rückschritte bei der Arbeitsgestaltung gegeben hat. In einigen Betrieben wurden auch die Ressourcen und Handlungsmöglichkeiten der Gruppen eingeschränkt: Die Zeitspielräume für Gruppengespräche sind geringer, die Personalplanung ist restriktiver geworden, die Gruppen haben weniger Möglichkeiten, auf betriebliche Planungsprozesse Einfluss zu nehmen.
Bezüglich der Entwicklung der betriebsorganisatorischen Strukturen registrieren die Betriebsräte bei vielen betrieblichen Führungskräften die Einschätzung, dass die funktionsübergreifende Zusammenarbeit und eine stärker an den jeweiligen Prozessbesonderheiten ausgerichtete Gestaltung von Abläufen insbesondere in größeren Betrieben nach wie vor nicht befriedigend gelöst sind. Konzepte einer stärker marktförmigen Ausgestaltung der Organisationsstrukturen und internen Kooperationsbeziehungen, die vielfach seit Mitte der 1990er Jahre eingesetzt wurden, befinden sich eher auf dem Rückzug. Aufwind haben dagegen - nicht zuletzt als Folge des Aufbaus zusätzlicher Standorte im Zuge von Globalisierungsprozessen - eine stärkere Zentralisierung von Entscheidungswegen und unternehmensweite Formalisierung von Prozessen.

\subsection{AUSEINANDERSETZUNGEN UM PRODUKTIONSSYSTEME}

Die in der Branche geführte Debatte um Produktionssysteme ist der Versuch, verschiedene, mit dem Leitbild Toyota verknüpfte Elemente der Gestaltung von Produktions- und Arbeitsstrukturen innerhalb eines einheitlichen Rahmens einzuführen. Zugleich ist sie Ausdruck des in vielen Betrieben vorhandenen Unbehagens an dem seit den 1990er Jahren zu beobachtenden Nach- und Nebeneinander unterschiedlicher und teilweise wenig kompatibler Reorganisationskonzepte. Jenseits allgemein gehaltener Prinzipien findet sich allerdings auch bei der Gestaltung von Produktionssystemen, der Auslegung der jeweiligen Einführungsprozesse und mit Blick auf die Rolle der betrieblichen Interessenvertretung hierbei eine erhebliche Spannbreite. Auf der einen Seite gibt es betriebliche Konstellationen, in denen Management und Betriebsräte die Realisierung eines Produktionssystems als gemeinsame Aufgabe betrachten und die aktive Beteiligung der Betriebsräte auch aus Sicht des Managements wichtig ist für die Qualität des Einführungsprozesses. Dies gilt auch in solchen Fällen, in denen beide Seiten davon ausgehen, dass es unterschiedliche Interessen gibt und die arbeitspolitischen Ziele keineswegs deckungsgleich sind. Es finden sich andererseits jedoch auch Beispiele für rein legitimatorische Beteiligungsangebote aus Gründen der Akzeptanzbeschaffung und Fälle, in denen die betriebliche Inte- ressenvertretung die Einführung von Produktionssystemen eher passiv beobachtend begleitet oder sich ausschließlich beteiligt, mit dem Ziel, Kontrollmöglichkeiten über arbeitspolitische Prozesse nicht ganz aus der Hand zu geben.

Auch wenn angesichts der Krise das Thema Beschäftigungssicherung alle anderen Politikbereiche überschattet, sind insbesondere die Betriebsräte in der Automobilindustrie in den bisher genannten Feldern seit Jahren aktiv und verfügen über ein erhebliches Maß an Wissen und Politikerfahrung.

\subsection{ARBEITSPOLITIK FÜR ANGESTELLTE ALS LEERSTELLE}

Ganz anders sieht es aus Sicht der Betriebsräte im Politikfeld „Arbeitspolitik für Angestellte" aus. Bezogen auf die Regulierung ausufernder Arbeitszeiten gibt es hier zwar erste Erfahrungen. Betrieblich erprobte Konzepte, wie sich die seit Jahren zunehmenden leistungspolitischen Probleme dieser Beschäftigtengruppe eindämmen lassen, sind aus Sicht der Betriebsräte aber Mangelware. Arbeitspolitisch, d.h. hinsichtlich der Gestaltung der Arbeits- und Organisationsstrukturen im Angestelltenbereich, fallen die Einschätzungen der Betriebsräte noch drastischer aus. Dieses Thema steht nur in Ausnahmefällen auf der Agenda, obwohl fast alle Befragten erheblichen Handlungsbedarf artikulieren: Die Umgestaltung von Arbeitsabläufen der Angestellten ist seit Jahren in vollem Gang. Zudem haben inhaltliche Aspekte von Arbeit, professionelle Arbeitsorientierungen sowie berufliche Entwicklungschancen gerade bei Angestellten einen hohen Stellenwert. ${ }^{4}$ Doch anders als für gewerbliche Tätigkeiten fehlen gerade in diesem quantitativ größer werdenden Bereich in der gewerkschaftlichen Debatte konkrete Leitbilder guter Arbeit, von denen her sich betriebliche Entwicklungen kritisieren und eigene Gestaltungskonzepte begründen ließen.

\footnotetext{
4 Eine wichtige Rolle spielt hierbei die Statusgruppe der ersten Führungsebene (häufig: Meister), die traditionell in besonders großem Umfang gewerkschaftlich organisiert war, die in besonderer Weise direkt im Fokus betrieblicher Reorganisationskonzepte steht oder hiervon zumindest betroffen wird und der zudem für das arbeitspolitische Klima in den Betrieben eine große Bedeutung zukommt.
} 


\subsection{BAUSTELLE KOORDINATIONS UND STEUERUNGSFORMEN}

Als unterausgeschöpft gilt auch das arbeitspolitische Handlungsfeld der Steuerungs- und Koordinationsformen, und das, obwohl im Rahmen der Vermarktlichungsdebatte vielfach auf den Wandel betrieblicher Steuerungsformen hingewiesen wurde und finanzorientierte Kennzahlen bis auf die betriebliche Ebene hinunter an Bedeutung zugenommen haben. Die Ausgestaltung und der betriebliche Umgang mit Kennzahlsystemen vollziehen sich weitgehend außerhalb der Einflusssphäre und teilweise auch der Aufmerksamkeit der betrieblichen Interessenvertretungen (Wagner 2005). Lediglich dort, wo Kennzahlen zum Bestandteil von Entgeltsystemen werden, sind sie Gegenstand von Interessenhandeln, eine Tendenz, die bisher trotz der reformierten Entgeltrahmentarifverträge (ERA) - aber noch wenig verbreitet ist. Auch für die Gestaltung betrieblicher Steuerungs- und Koordinationsformen gilt, dass sie zukünftig sehr viel stärker als arbeitspolitisches Handlungsfeld $\mathrm{zu}$ entdecken wäre: unter dem Gesichtspunkt der Entwicklung eigenständiger leistungspolitischer Konzepte, aber auch mit Blick auf die Verbesserung betrieblicher Kooperationsstrukturen sowie vor dem Hintergrund der Frage, welchen Stellenwert finanzbezogene Kenngrößen im Arbeitshandeln von verschiedenen Funktionsbereichen und Tätigkeitsfeldern haben (sollten).

\subsection{ARBEITSFORMEN DER INTERES- SENVERTRETUNG: AUSSCHUSS- VERSUS PROJEKTARBEIT?}

Schließlich registrieren die von uns befragten Betriebsräte beim Thema Arbeitspolitik auch veränderte Anforderungen an die Arbeitsweise der betrieblichen Interessenvertretung. Arbeitspolitische Themen haben in den Betriebsratgremien einen sehr unterschiedlichen Stellenwert. Fast durchweg gibt es in den Interessenvertretungen zwar einzelne Personen, die die Arbeitsund Organisationsgestaltung $\mathrm{zu}$ ihrem Themenschwerpunkt gemacht haben; nur in einem Teil der Betriebe existieren jedoch auch eigenständige Gremien (Ausschüsse, Arbeitsgruppen), die eine kontinuierliche Bearbeitung arbeitspolitischer Fragestellungen gewährleisten. In manchen Betrieben liegt die arbeitspolitische Arbeit ausschließlich bei temporären Projektgrup- pen, die sich entlang von Managementprojekten - in einzelnen Fällen auch gewerkschaftlichen Kampagnen - bilden. Die von uns befragten Betriebsräte registrieren Defizite in Bezug auf das Rollenverständnis und die Kompetenzen der betrieblichen Interessenvertretung, auf die zumeist wenig konsolidierte und häufig von punktuellen Aktivitäten geprägte Gremienlandschaft sowie im Bereich der überbetrieblichen Vernetzung.

Mit Blick auf das Rollenverständnis artikulieren Betriebsräte einerseits eine besonders deutliche Kritik an der nach wie vor verbreiteten Stellvertreterorientierung in der Interessenvertretungsarbeit. Die viel diskutierte Beteiligung der Beschäftigten ist in der Praxis immer noch sehr begrenzt. Andererseits sehen Betriebsräte aber auch, dass eine weitergehende Professionalisierung für eine effektive Interessenvertretung unverzichtbar ist. Insbesondere gegenüber dem Management erfordert Arbeitspolitik sowohl umfängliches Expertenwissen bezogen auf arbeits- und betriebsorganisatorische Sachthemen als auch systematisches Know-how im Bereich Organisationsentwicklung. Mit dem Hinweis auf beteiligungsorientierte Interessenvertretungsarbeit ist es folglich nicht getan. Notwendig sind zugleich fundierte Sachkenntnisse und ein hohes Maß an Professionalität und Expertenwissen im Bereich der Arbeitsund Organisationsgestaltung.

Eine Diskussion über geeignete Arbeitsformen im Handlungsfeld Arbeitspolitik hat noch nicht wirklich begonnen, spielt in den Überlegungen einzelner Betriebsräte jedoch eine erhebliche Rolle. Die formelle Berücksichtigung des Themas durch einen eigenständigen Ausschuss wird als sinnvoll erachtet, ${ }^{5}$ zugleich jedoch die Gefahr gesehen, dass die enge Verschränkung arbeitspolitischer Themen mit anderen Handlungsfeldern (insbesondere Leistungspolitik, Arbeitsschutz und Qualifizierung) hierdurch aus dem Blick geraten könnte. Arbeitspolitik wird im Kern als projektförmig zu bearbeitende Querschnittsaufgabe betrachtet, das Verhältnis von Gremien- und Projektarbeit gilt vielfach als noch klärungsbedürftig.

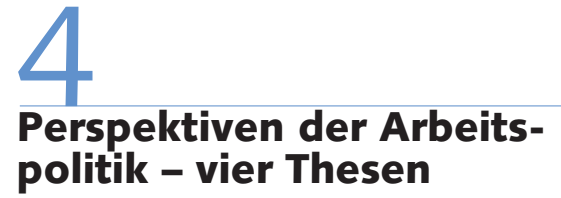

(1) Auch für den Fall, dass es als Folge der Finanzmarktkrise zu Veränderungen auf der Ebene der Corporate Governance kommt, spricht derzeit wenig dafür, dass sich Problemlagen und Handlungsbedingungen im Politikfeld Arbeitspolitik wesentlich verändern. Da allenfalls von einem Verhältnis loser Kopplung zwischen Corporate Governance und Arbeitspolitik auszugehen ist und den betrieblichen Akteuren erhebliche Spielräume in der Übersetzung von Rahmenbedingungen in betriebliche Politiken bleiben, dürften von einem Wandel auf der regulativen Ebene zumindest keine starken und eindeutigen Impulse für Arbeitspolitik ausgehen (vgl. hierzu auch Dörre/Holst in diesem Heft). Kostendruck und Renditeerwartungen bleiben angesichts globalisierter Produktmärkte und neuer Akteure auf dem Weltmarkt weiterhin hoch, und es gibt bisher keine Hinweise, dass es zu einer Umorientierung bei den arbeitspolitischen Leitbildern kommt.

(2) Derzeit spricht mehr für ein Fortbestehen des spannungsgeladenen Nebeneinanders divergierender und häufig gegensätzlich ausgerichteter Trends. Dies bedeutet aber zugleich, dass die vorhandenen Gestaltungsspielräume für arbeitspolitische Initiativen genutzt werden können. Die Frage, wie sich Wirtschaftlichkeits- und Arbeitsverbesserungen kombinieren lassen, hat für arbeitspolitische Initiativen weiterhin eine große Bedeutung. Wirtschaftlichkeitsüberlegungen (Produktivität, Qualität, Effektivität und Innovationsfähigkeit) lassen sich nicht auf finanzökonomische

\footnotetext{
5 Argumente für eigenständige Gremien sind, dass hierdurch eine größere Kontinuität und Sichtbarkeit beim Thema Arbeitspolitik erreicht wird und dass kontinuierliche Gremienarbeit zugleich eine Grundlage für notwendige Professionalisierungsprozesse bildet. Insbesondere gegenüber den auch im Management nicht selten stark arbeitsteiligen Strukturen würde eine Bündelung der verschiedenen Facetten durch die Interessenvertretung einen Vorteil bedeuten. Zudem könnte die von vielen Betriebsräten beklagte mangelhafte überbetriebliche Vernetzung - sowohl innerhalb der jeweiligen Unternehmen, aber auch mit anderen Unternehmen sowie mit gewerkschaftlichen Initiativen - von der Institutionalisierung eigenständiger Gremien profitieren.
} 
Anforderungen reduzieren und sind von Nachhaltigkeitsfragen nicht zu trennen. Ausgehend von Maßstäben "guter Arbeit" geht es darum, eine Auseinandersetzung über arbeitspolitische Leitbilder zu führen, für die sich vor dem Hintergrund der mittlerweile wiederbelebten Debatte über Wirtschaftsdemokratie und Unternehmensmitbestimmung neue Bezugspunkte ergeben (Demirović 2007; Schumann 2007).

(3) Voraussetzung für eine Revitalisierung der Arbeitspolitik ist ein erweiterter Blick auf die Gestaltung betrieblicher Strukturen aus einer doppelten Perspektive: „Halten und Entfalten“. Erfolgreich - auch im
Sinne von auf Dauer durchsetzungsfähig ist eine innovative Arbeitspolitik nur dann, wenn sie in ihrer Gestaltungstiefe und -reichweite über die bisherigen Ansatzpunkte hinausgeht und bezogen auf unterschiedliche Handlungsfelder konkrete Gestaltungskonzepte entwickelt. So sind über die Ebene der Arbeitsorganisation hinaus insbesondere betriebsorganisatorische Strukturen, Steuerungs- und Koordinationsformen, Fragen der betrieblichen Führung und Formen der Produkt- und Prozessoptimierung in den Blick zu nehmen. Für die betrieblichen Interessenvertretungen geht es zum einen darum, Retaylorisierungs-, Vermarktlichungs- und
Prekarisierungstendenzen zu begrenzen („Halten“). Zugleich sind sie gefordert, die sozialen und wirtschaftlichen Potenziale qualifizierter, beteiligungsintensiver Arbeitsstrukturen sowie funktions- und hierarchieübergreifender Kooperationsprozesse offensiver auszureizen („Entfalten“).

(4) Aus den Gesprächen mit Betriebsräten ist deutlich geworden, dass eine Perspektive der Neubelebung von Arbeitspolitik auch Folgen haben dürfte für die Rollendefinitionen und Arbeitsweisen der betrieblichen Interessenvertretungen. Die Diskussion hierüber hat jedoch gerade erst begonnen.

\section{LITERATUR}

Baethge, M. (1991): Arbeit, Vergesellschaftung, Identität - Zur zunehmenden normativen Subjektivierung der Arbeit, in: Soziale Welt 1, S. 6-19 Brinkmann, U./Dörre, K./Röbenack, S./Kraemer, K./Speidel, F. (2006): Prekäre Arbeit. Ursachen, Ausmaß, soziale Folgen und subjektive Verarbeitungsformen unsicherer Beschäftigungsverhältnisse, Bonn

Castel, R./Dörre, K. (Hrsg.) (2009): Prekarität, Abstieg, Ausgrenzung. Die soziale Frage am Beginn des 21. Jahrhunderts, Frankfurt/Main Demirović, A. (2007): Demokratie in der Wirtschaft. Positionen - Probleme - Perspektiven, Münster

Detje, R./Pickshaus, K./Urban, H.-J. (Hrsg.) (2005): Arbeitspolitik kontrovers. Zwischen Abwehrkämpfen und Offensivstrategien, Hamburg Dörre, K. (2001a): Das deutsche Produktionsmodell unter dem Druck des Shareholder Value, in: Kölner Zeitschrift für Soziologie und Sozialpsychologie 4, S. 675-704

Dörre, K. (2001b): Das Pendel schwingt zurück. Arbeit und Arbeitspolitik im flexiblen Kapitalismus, in: Ehlscheid, C./Mathes, H./Scherbaum, M. (Hrsg.): „Das regelt schon der Markt!“ Marktsteuerung und Alternativkonzepte in der Leistungs- und Arbeitszeitpolitik, Hamburg, S. 37-58 Dörre, K. (2005): Prekarität. Eine arbeitspolitische Herausforderung, in: WSI-Mitteilungen 5, S. 250-258

Dörre, K./Röttger, B. (Hrsg.) (2003): Das neue Marktregime. Konturen eines nachfordistischen Produktionsmodells, Hamburg

Ezzamel, M./Willmott, H./Worthington, F. (2008): Manufacturing shareholder value: The role of accounting in organizational transformation, in: Accounting, Organizations and Society 2, S. 107-140

Froud, J./Johal, S./Leaver, A./Williams, K. (2006): Financialization and Strategy. Narrative and Numbers, London

Gerst, D. (1998): Selbstorganisierte Gruppenarbeit - Gestaltungschancen und Umsetzungsprobleme, Eschborn

Gryglewski, S. (2007): Sicherung von Produktionsarbeit in Deutschland. Reformbedarf der arbeitsorganisatorischen Leitbilder, in: Zeitschrift für Arbeitswissenschaft 1, S. 47-53

Haipeter, T. (2009): Tarifabweichungen und Flächentarifverträge. Eine Analyse der Regulierungspraxis in der Metall- und Elektroindustrie, Wiesbaden
Höpner, M. (2003): Wer beherrscht die Unternehmen? Shareholder Value, Managerherrschaft und Mitbestimmung in Deutschland, Frankfurt/Main

IG Metall (Hrsg.) (2001): Flexible Standardisierung, Frankfurt/Main Jürgens, U. (1997): Rolling Back Cycle Times: The Renaissance of the Classical Assembly Line in Final Assembly, in: Shimokawa, K./Jürgens, U./Fujimoto, T. (Hrsg.): Transforming Automobile Assembly, Berlin, S. $255-273$

Jürgens, U. (2007): Warum Toyota so lange so stark ist, Stuttgart Jürgens, U./Naschold, F. (1994): Arbeits- und industriepolitische Entwicklungsengpässe der deutschen Industrie in den neunziger Jahren, in: Zapf, W./Dierkes, M. (Hrsg.): Institutionenvergleich und Institutionendynamik, Berlin, S. 239-270

Kern, H./Sabel, C. (1994): Verblaßte Tugenden. Zur Krise des deutschen Produktionsmodells, in: Beckenbach, N./van Treeck, W. (Hrsg.): Umbrüche gesellschaftlicher Arbeit, Soziale Welt, Sonderband 9, Göttingen, S. 605-624

Kern, H./Schumann, M. (1984): Das Ende der Arbeitsteilung? Rationalisierung in der industriellen Produktion, München

Kleemann, F./Matuschek, I./Voß, G. (2003): Subjektivierung von Arbeit. Ein Überblick zum Stand der Diskussion, in: Moldaschl, M./Voß, G. (Hrsg.): Subjektivierung von Arbeit, München und Mering, S. 57-114 Kotthoff, H./Wagner, A. (Hrsg.) (2008): Die Leistungsträger, Berlin Kratzer, N./Menz, W./Nies, S./Sauer, D. (2008): Leistungspolitik als Feld "umkämpfter Arbeit“, in: Prokla 150, S. 11-26

Kuhlmann, M. (2004): Modellwechsel? Die Entwicklung betrieblicher Arbeits- und Sozialstrukturen in der deutschen Automobilindustrie, Berlin Kuhlmann, M. (2007): Jenseits von Gruppenarbeit - Perspektiven der Arbeitspolitik, in: Peter, G. (Hrsg.): Grenzkonflikte der Arbeit, Hamburg, S. 129-143

Kuhlmann, M./Schumann, M. (2000): Was bleibt von der Arbeitersolidarität? Zum Arbeits- und Betriebsverständnis bei innovativer Arbeitspolitik, in: WSI-Mitteilungen 1, S. 18-27 
Kuhlmann, M./Sperling, H. J./Balzert, S. (2004): Konzepte innovativer Arbeitspolitik. Good-Practice-Beispiele aus dem Maschinenbau, der Automobil-, Elektro- und Chemischen Industrie, Berlin

Lehndorff, S. (Hrsg.) (2009): Abriss, Umbau, Renovierung? Studien zum Wandel des deutschen Kapitalismusmodells, Hamburg

Liker, J. K. (2004): The Toyota Way. 14 Management Principles from the World's greatest Manufacturer, New York

Lohr, K. (2003): Subjektivierung von Arbeit. Ausgangspunkt einer Neuorientierung der Industrie- und Arbeitssoziologie?, in: Berliner Journal für

Soziologie 4, S. 511-529

Moldaschl, M./Sauer, D. (2000): Internalisierung des Marktes - Zur neuen Dialektik von Kooperation und Herrschaft, in: Minssen, H. (Hrsg.): Begrenzte Entgrenzungen. Wandlungen von Organisation und Arbeit, Berlin, S. 205-224

Peters, K./Sauer, D. (2005): Indirekte Steuerung - eine neue Herrschaftsform. Zur revolutionären Qualität des gegenwärtigen Umbruchprozesses, in: Wagner, H. (Hrsg.): „Rentier' ich mich noch“? Neue Steuerungskonzepte im Betrieb, Hamburg, S. 23-58

Pickshaus, K./Urban, H.-J. (2009): Gute Arbeit in Krisenzeiten - Arbeitspolitik zwischen defensiver Krisenabwehr und offensiver Krisenüberwindung, in: WSI-Mitteilungen 6, S. 331-334

Pries, L./Schmidt, R./Trinczek, R. (1990): Entwicklungspfade von Industriearbeit, Opladen

Rehder, B. (2003): Betriebliche Bündnisse für Arbeit in Deutschland, Frankfurt/Main
Schumann, M. (1998): Frißt die Shareholder Value-Ökonomie die Modernisierung der Arbeit?, in: Hirsch-Kreinsen, H./Wolf, H. (Hrsg.): Arbeit,

Gesellschaft, Kritik, Berlin, S. 19-30

Schumann, M. (2007): Warum (gerade heute) Mitbestimmung? Zur Kontroverse um die Reform der deutschen Unternehmensmitbestimmung, in: Personalführung 3, S. 60-67

Schumann, M. (2008): Kampf um Rationalisierung - Suche nach neuer Übersichtlichkeit, in: WSI-Mitteilungen 7, S. 379-386

Schumann, M./Kuhlmann, M./Sanders, F./Sperling, H. J. (Hrsg.) (2006):

VW Auto 5000: ein neues Produktionskonzept. Die deutsche Antwort auf den Toyota-Weg?, Hamburg

Schumann, M./Kuhlmann, M./Sperling, H. J. (2008): Zwischen Toyota und Tradition: Das VW-Projekt "Auto 5000“ als mitbestimmungsjustierte Unternehmenskultur, in: Benthien, R./Brinkmann, U. (Hrsg.): Unternehmenskultur und Mitbestimmung, Frankfurt/Main, S. 243-258

Streeck, W. (2009): Re-Forming Capitalism, Oxford

Vester, M./Lange-Vester, A./Teiwes-Kügler, C. (2007): Die neuen Arbeitnehmer. Zunehmende Kompetenzen - wachsende Unsicherheit, Hamburg

Wagner, H. (Hrsg.) (2005): "Rentier' ich mich noch“? Neue Steuerungskonzepte im Betrieb, Hamburg

Windolf, P. (Hrsg.) (2005): Finanzmarkt-Kapitalismus, Kölner Zeitschrift für Soziologie und Sozialpsychologie (KZfSS), Sonderheft 45, Wiesbaden 